\title{
Programa de Educação Continuada Voltado ao Uso de Tecnologias em Saúde: Percepção dos Profissionais de Saúde
}

\author{
Continuing Education Program for the Use of Health Technologies: \\ Perception of Health Professionals
}

\author{
ROSANE BARRETO CARDOSO \\ SÉRGO BASSALO PALUDETO \\ BEATRIZ JASEN FERREIRA ${ }^{3}$
}

\section{RESUMO}

Objetivo: Identificar a percepção dos profissionais de saúde quanto a um programa de Educação Continuada (EC) voltada ao uso de novas tecnologias no campo da saúde. Materiais e Métodos: Estudo descritivo e exploratório de abordagem quantitativa, realizado em um hospital privado situado na cidade do Rio de Janeiro, Brasil. Para coleta de dados foi elaborado um instrumento de percepção do tipo atitudinal Likert, composto por quatro dimensões (serviço de EC como um espaço estimulador de conhecimento sobre Tecnologias de Informação e Comunicação (TIC) em saúde; uso de TIC em saúde como ferramenta estimuladora do trabalho interprofissional; uso do Prontuário Eletrônico do Paciente (PEP) e a sua relação com a promoção do processo de Educação Permanente em Saúde (EPS); e, o PEP como instrumento qualificador para assistência à saúde) e 17 asserções positivas randomizadas. Participaram do estudo 71 profissionais de saúde (médico, enfermagem e fonoaudiólogo). Resultados: A média da pontuação das dimensões estava dentro do intervalo de classificação denominado "percepção boa/positiva", o que indicou concordância entre os respondentes quanto às ações desenvolvidas pelo programa de EC. Os participantes inferiram o programa como um espaço estimulador para a qualificação de uso de tecnologias no processo de cuidar, trabalho interprofissional e promoção da EPS.Conclusão:As ações do programa de EC possuem potencial para qualificar a assistência à saúde e a construção de significados relacionados ao uso de novas tecnologias em saúde.

\section{DESCRITORES}

Educação Permanente.Educação em Saúde.Tecnologia da Informação.

\begin{abstract}
Objective: To identify the perception of health professionals regarding a Continuing Education (CE) program for the use of new technologies in health. Materials and Methods: This is adescriptive and exploratory study with a quantitative approach, carried out in a private hospital located in the city of Rio de Janeiro, Brazil. The Likert's attitude scale was used in this study. This tool is composed of four dimensions (CE service as a way to encourage knowledge acquisition on Information and Communication Technologies (ICT) in health; use of health ICT as a stimulating tool for multidisciplinary work; use of the Electronic Patient Record (EPR) and its relation with the Permanent Health Education (PHE); and the EPR as a qualifier tool for health care) and composed of 17 randomized positive assertions. A total of 71 health professionals (physicians, nurses and speech therapists) participated in the study. Results: The average score obtained was within the category "good/positive perception", which indicated agreement among respondents about the actions taken by the CE program. Participants inferred the program as a stimulating space for the qualification of the use of technologies in the care process, multidisciplinary work and promotion of PHE. Conclusion: The CE program actions have the potential to qualify health care and the construction of meanings related to the use of new health technologies.
\end{abstract}

\section{DESCRIPTORS}

Continuing Education.Health Education.Information Technology.

Enfermeira de Desenvolvimento Operacional e Administrativo, Hospital Unimed Rio. Rio de Janeiro. Rio de Janeiro. Brasil.

2 Coordenador de Tecnologia da Informação, Hospital Unimed Rio. Rio de Janeiro. Rio de Janeiro. Brasil.

3 Docente do Programa de Pós Graduação Ensino em Ciências da Saúde do Centro de Desenvolvimento do Ensino Superior em Saúde - CEDESS. Universidade Federal de São Paulo - UNIFESP. São Paulo. São Paulo. Brasil. 
A educação continuada (EC) é uma necessidade premente nas instituições frente às exigências de mercado e das mudanças decorrentes do uso de novas tecnologias em saúde. As ações desenvolvidas pela EC podem aproximar a lacuna existente entre a formação e a real necessidade das instituições ${ }^{1,2}$.

A EC surgiu como uma estratégia para a capacitação de profissionais da saúde já inseridos no serviço. A EC pode ser definida como um conjunto de atividades educativas que visam à propagação de conhecimentos, práticas e reflexões do processo de trabalho ${ }^{3,4}$.

A EC está atrelada a educação permanente em saúde (EPS), favorecendo a construção de novos saberes e reflexão da prática. Uma ferramenta com grande potencial para a disseminação do uso seguro de tecnologias no campo da saúde ${ }^{3-5}$.

As Tecnologias de Informação e Comunicação (TIC) deram um novo rumo à forma de comunicação em todas as áreas do conhecimento. A tecnologia passou a desempenhar um papel importante na estrutura organizacional da sociedade permitindo o processamento, o armazenamento, a difusão da informação e do conhecimento ${ }^{2,6}$

No âmbito da saúde as TIC têm apoiado a organização e gestão dos processos institucionais, integrando os recursos computacionais à prática profissional ${ }^{7,8}$.

O prontuário eletrônico do paciente (PEP) é uma das TIC que as instituições de saúde têm adotado para a gestão de seus processos de trabalho a fim de alcançar maior produtividade, tornando as informações mais disponíveis, facilitando a comunicação e aprimorando a qualidade da assistência prestada ao paciente $e^{9,10}$.

O desenvolvimento e uso de tecnologias trouxe algumas repercussões para o trabalho dos profissionais da saúde, diante do redimensionamento do espaço assistencial. A equipe de saúde passou a ter que assistir o paciente ao mesmo tempo que dominar os vários tipos de tecnologia ${ }^{10}$, 11.

Contudo, o paradigma da introdução das TIC no processo de cuidar não reside somente no estabelecimento de como manuseá-las, mas também em traçar estratégias para melhor conhecêlas e obter qualidade do cuidado prestado produzido por elas.

Estabelecer um programa de EC tendo como base a interdisciplinaridade propiciará a interação da equipe de saúde ${ }^{6}$. Assim, como poderá oportunizar a compreensão do uso da tecnologia como ferramenta de trabalho para uma ação transformadora do cuidado humano.

Neste sentido torna-se essencial implementar programas de EC voltado ao desenvolvimento do uso de novas tecnologias no campo da saúde, à luz de um processo de aprendizagem significativa no trabalho. Este estudo teve como objetivo identificar a percepção dos profissionais de saúde quanto a um programa de EC voltada ao uso de novas tecnologias no campo da saúde.

\section{MATERIAL E MÉTODOS}

Trata-se de um estudo descritivo e exploratório de abordagem quantitativa, realizado com profissionais de saúde atuantes em um hospital privado localizado na cidade do Rio de Janeiro/RJ, Brasil.

O cenário do estudo foi um hospital privado de nível terciário, inaugurado no mês de janeiro de 2013. O hospital possui 213 leitos dentre os quais 132 são destinados à unidade de internação clínica e cirúrgica, e 81 são destinados ao centro de terapia intensiva e 11 salas de cirurgias.

Para compor a população do estudo foi adotado como critério de inclusão: profissionais de saúde que participaram da capacitação referente ao uso do PEP na instituição, no período de junho a julho de 2014.

Para a coleta de dados foi elaborado um instrumento de percepção do tipo atitudinal Likert. Este foi disponibilizado no formato impresso e eletrônico, por meio do aplicativo do Google Forms, sendo oferecido aos 160 profissionais de saúde que participaram da capacitação.

A coleta de dados foi realizada no período de setembro a outubro de 2014. Participaram 71 respondentes que retornaram com o questionário preenchido adequadamente.

O instrumento de pesquisa proposto para coleta de dados foi composto por quadro dimensões (D), a saber: D1, serviço de EC como um espaço estimulador de conhecimento sobre TIC em saúde; D2, o uso de TIC em saúde como ferramenta estimuladora do trabalho interprofissional; D3, o uso do PEP e a sua relação com a promoção do processo de EPS; e D4, o PEP como instrumento qualificador para assistência à saúde.

As dimensões e suas respectivas asserções foram elaboradas com base na experiência dos autores, que participaram de 
implantação e treinamentos relacionados à TIC em saúde em hospitais no Brasil, assim como na literatura atual sobre EPS, EC e uso de tecnologias em saúde.

Para cada dimensão foram elaboradas de três a sete asserções $(A)$ que no final consistiram em 17 assertivas positivas randomizadas. As asserções buscaram identificar a percepção dos respondentes sobre o objeto pesquisado. Os respondentes puderam escolher dentre quatro opções de resposta: concordo plenamente (CP), inclinado a concordar (IC), inclinado a discordar (ID) e discordo plenamente (DP). Optou-se por um número par de escolhas de resposta para cada asserção visando evitar uma tendência centrante.

Para cada uma das opções de resposta foi atribuída previamente uma pontuação entre 1 (mínimo) e 4 (máximo) pontos. Tomando por base que as pontuações das assertivas e das dimensões poderiam variar de 1 a 4 pontos, os resultados das médias foram agrupados da seguinte forma: de 1,00 a 1,99 (percepção ruim/negativa); de 2,00 a 2,99 (percepção intermediária), e de 3,00 a 4,00 (percepção boa/positiva).

Também foram elaboradas questões fechadas no intuito de obter informações para conhecer a população do estudo, tais como: sexo, com duas opções de resposta (feminino e masculino); faixa etária, com a composição de quatro grupos (18 a 25 anos, 26 a 35 anos, 36 a 45 anos e $>45$ anos); formação profissional, dividido em quatro alternativas (enfermeiro, médico, técnico em enfermagem ou outros); tempo de formação, com quatro preferências de resposta ( $<1$ ano, 1 ano a 4 anos, 5 anos a 10 anos e >10 anos); nível de conhecimento em informática, com três seleções de respostas (básico, intermediário e avançado); estímulo ao uso de TIC na graduação/formação e experiência anterior com PEP, estas últimas com duas opções de resposta (sim ou não).

Foi realizado o pré-teste do instrumento antes de ser aplicado à população delimitada.

$\mathrm{O}$ instrumento foi submetido à confiabilidade de coerênciainternasendo calculada por

\footnotetext{
Quadro 1. Dimensões do Instrumento Likert e suas respectivas asserções, Rio de Janeiro, Brasil.

A1 A introdução de treinamentos relacionados ao uso de TIC em saúde é importante para o proarama de EC da instituicão.

A3 No programa de treinamento admissional é essencial à capacitação sobre a utilização do PEP.

A5 O serviço de EC contribui para o desenvolvimento de competências, habilidades e saberes específicos em TIC em saúde.

$\bar{\square}$

A7 A capacitação referente ao PEP contribui para o fortalecimento do trabalho coletivo da equipe interprofissional em saúde.

A9 A estratégia de ensino utilizada no treinamento favoreceu adequadamente o aprendizado.

A11 A carqa horária estipulada para a capacitacão de uso do PEP foi adequada.

A13 O profissional que efetuou a capacitação de utilização do PEP estava preparado para o A13 desenvolvimento desta atividade.

A2 O PEP é uma ferramenta informatizada que facilita a prática da assistência interprofissional.

₹ A4 A capacitação sobre o PEP oferecida pela instituição facilitou a compreensão do A4 processo de trabalho da equipe interprofissional.

A6 O PEP facilita a busca de informações do paciente, facilitando a tomada de decisão da A6 equipe interprofissional.

A10 O uso do PEP contribui para o melhor uso de outros tipos de TIC.

m A14 A utilização do PEP facilita a promoção do processo de EPS na instituição.

A16 O PEP proporciona a interação da equipe interprofissional com a troca de experiências, A16 promovendo a EPS.

A12 O PEP viabiliza a orqanização da assistência prestada aos pacientes.

\& A15 A utilizacão do PEP contribuipara a reducão de possíveis erros assistenciais.

A17 O PEP favorece a compreensão e interação das ações assistenciais, propostas pela equipe interprofissional.
} 
meio do teste de coeficiente alfa de Cronbach. O teste mede a correlação entre as respostas do questionário determinadas pelos respondentes, apresentando uma correlação média entre as perguntas $^{12,13}$. A probabilidade de erro foi estabelecida em $5 \%$.

O projeto desta investigação foi submetido à apreciação do Comitê de Ética em Pesquisa da Universidade Federal de São Paulo, e recebeu parecer favorável sob o número 30221414.6.0000.5505.

Os dados foram armazenados em planilha eletrônica Exce/® 2010 (Microsoft) e analisados por meio do processamento dos recursos de computação do sistema StatisticPackage for Social Sciences (SPSS).

\section{RESULTADOS}

Dentre os 160 questionários enviados $71(44,3 \%)$ profissionais de saúde aceitaram participar da pesquisa e responderam o questionário adequadamente. Entre os respondentes $36(50,70 \%)$ eram enfermeiros, $30(42,25 \%)$ médicos, $4(5,63 \%)$ técnicos em enfermagem e $1(1,42 \%)$ fonoaudiólogo. Dentre estes profissionais, $12(16,90 \%)$ eram homens e 59(83,10\%) eram mulheres.

Quanto à faixa etária, $8(11,27 \%)$ pertenciam ao primeiro grupo com idade até 25 anos, $38(53,52 \%)$ estavam entre 26 e 35 anos, $16(22,54 \%)$ entre 36 e 45 anos e $9(12,67 \%)$ pertenciam ao grupo com idade $>45$ anos.

Referente ao tempo de formação acadêmica, $3(4,22 \%)$ profissionais eram recémformados (>de 1 ano), 24(33,80\%) entre1 a 4 anos de formação/graduação, 22(30,99\%) entre 5 anos a 10 anos e $22(30,99 \%)>10$ anos.

Quanto ao nível de conhecimento em informática, 26(36,62\%) profissionais relataram possuir nível básico, 35(49,30\%) nível intermediário e 10(14,08\%) nível avançado. Dos 71 participantes $31(43,66 \%)$ profissionais relataram já possuir experiência com PEP e 14(19,72\%) profissionais afirmaram que na graduação/formação tiveram alguma disciplina ou estímulo ao uso de TIC.

O teste de confiabilidade do instrumento resultou em um coeficiente igual a $0,82(82 \%)$. O número de asserções validadas foram 16(94,12\%) com perda de 1(5,88\%) asserção pertencente àD1 .

As Figuras 1 e 2 apresentam a médiados pontos da escala Likert obtidos pelos profissionais por dimensão e asserção.

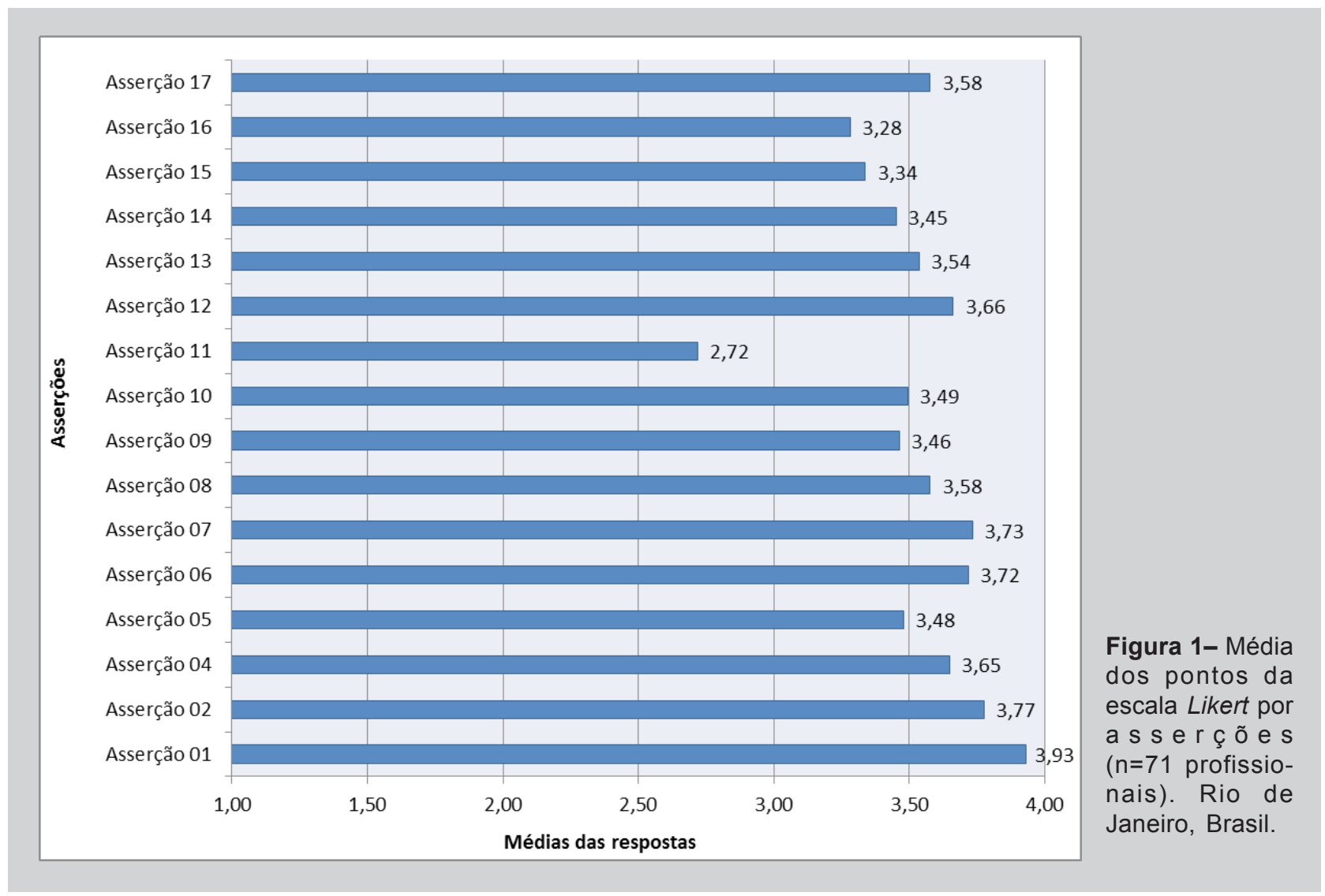




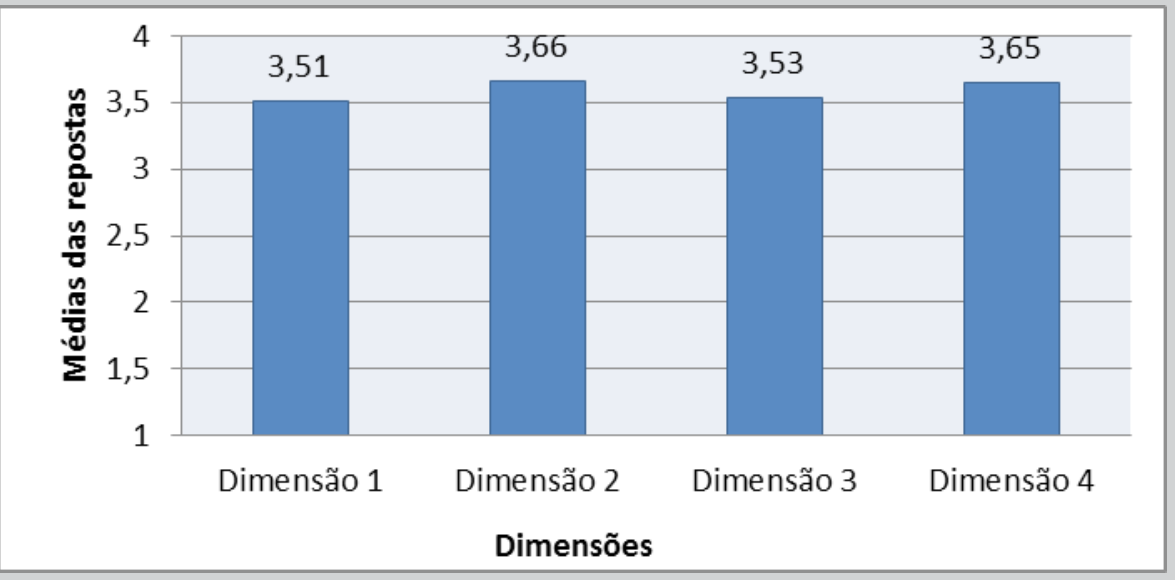

Figura 2 - Média dos pontos da escala Likertpor dimensão ( $n=71$ profissionais). Rio de Janeiro, Brasil.

\section{DISCUSSÃO}

O resultado de confiabilidade interna do instrumento Likert foi de 0,82(82\%), indicando um bom nível de confiança. O valor mínimo aceitável para a confiabilidade de um questionário é $\alpha \geq$ $0,70(70 \%)$, abaixo desse valor a consistência interna é considerada baixa ${ }^{12,13}$. O resultado do teste também denota que os itens do instrumento são homogêneos e que a escala mede consistentemente a característica para a qual foicriada ${ }^{13}$.

Para escala atitudinal Likert considera-se adequada a perda de até 30 a $40 \%$ do total de assertivas $^{12,13}$. Neste estudo a perda foi de apenas uma assertiva $(5,88 \%)$, isto reafirma a qualidade da validade de conteúdo ${ }^{12,13}$, considerando aspectos como adequação da fraseologia, objetividade e assertividade nas proposições apresentadas.

De modo geral, a média da pontuação das asserções validadas estava dentro do intervalo de classificação denominado "percepção boa/positiva", o que indica concordância entre os respondentes quanto as ações do programa de EC. Observamse comportamentos diferenciados dos profissionais em poucos itens, que foram discutidos abaixo.

A D1buscou observar a percepção dos profissionais quanto à identificação das atividades desenvolvidas pelo serviço de EC como um espaço estimulador de conhecimento sobre TIC em saúde. Nesta dimensão, nas asserções A1, A5, A7, A9 e A13 os respondentes ficaram inclinados a concordar, atribuindo ao serviço um espaço importante para a qualificação do uso das TIC em saúde.

Em todas as áreas do conhecimento, a busca pelo processo educativo que acompanhem os profissionais e promova transformação no ambiente de trabalho tem sido constante. As instituições de saúde vêm incorporando treinamentos e capacitações continuadamente, reconhecendo a importância do processo de aprendizagem no ambiente de trabalho para manter a qualidade da assistência prestada aos seus pacientes $5,14,15$. A formação continuada dos profissionais motiva a busca contígua por novos saberes, assim como a inserção de uma aprendizagem baseada em novas tecnologias ${ }^{5}$.

A educação permanente, continuada e em serviço, são estratégias desenvolvidas nas organizações que contribuem para a transformação pessoal e profissional. São ações e práticas educativas com foco na resolução de problemas, alternativas para minimizar dificuldades no trabalho como a utilização de TIC no processo de cuidar ${ }^{4,5}$.

$\mathrm{Na}$ D1, a asserção 3,obteve média de 3,99 pontos. Essa pontuação evidenciou um consenso positivo entre os respondentes, de tal modo resultou na perda da asserção. Neste caso não houve dispersão estatística adequada para esta asserção, contudo esta concordância revelou entre os respondentes a importância da capacitação do uso PEP como elemento potencial para a assistência.

$\mathrm{Na}$ literatura $\mathrm{a}^{8-11}$ há evidências que a utilização do PEP nas instituições de saúde pode trazer significativas vantagens para a assistência. 
Pois, o PEP contribui para a qualidade das informações do paciente, dados importantes e essenciais tanto para continuidade da assistência como para planejamento das ações gerenciais.

Na D1, a asserção11, que mencionava a carga horária da capacitação de uso do PEP,foi avaliada pelos profissionais de maneira negativa. Nesta asserção os respondentes mostram-se inclinados a discordar com 2,84 pontos. Essa pontuação norteou as ações do serviço de EC da instituição, no sentido de ampliar o tempo da formação ofertada em capacitações e treinamentos referentes ao PEP. Após, o resultado da pesquisa foi ampliada a carga horária da capacitação do uso do PEP para mais 4 horas, totalizando 8 horas.

Ainda na $\mathrm{D} 1$, ao analisar os resultados segundo o sexo, faixa etária, categoria profissional, tempo de formação, nível de conhecimento em informática, antecedente de uso do PEP e estímulo ao uso de TIC na graduação/formação, observouse que a média dos pontos foi convergente apontando uma percepção positiva entre os respondentes. É importante ponderar que se espera haver compreensões diferentes entre os sexos, as mulheres geralmente são mais orientadas por aspectos interpessoais e comportamentos expressivos, enquanto que homens são mais orientados pelo desempenho ${ }^{16}$.

Vale ressaltar que na D1 os respondentes com conhecimento básico em informática foram mais positivos, em relação aos profissionais que declararam nível intermediário e avançado. Os participantes que relataram estímulo ao uso de TIC na formação/graduação, também apontaram uma "percepção boa/positiva".

A incorporação de TIC na formação dos profissionais de forma a estabelecer relações com a prática na saúde, contribui no processo educativo e na concepção do aprendiz quanto ao uso de tecnologias na sua atuação profissional ${ }^{17,18}$.

A D2 tratou da percepção dos profissionais de saúde quanto ao uso de TIC como ferramenta estimuladora de qualidade da assistência á saúde numa perspectiva de exercício interprofissional. Os respondentes tiveram uma percepção positiva em todas as asserções (A2, A4, A6 e A8), afirmando que o uso de TIC em saúde é concebido pelos envolvidos como uma ferramenta estimuladora para o trabalho interprofissional.

As TIC constituem-se em um importante insumo estratégico institucional. A tecnologia é capaz de influenciar o negócio da empresa e desenvolver novas relações entre as pessoas, um exponencial para o estímulo do trabalho interprofissional ${ }^{18,19}$. Uma perspectiva de interprofissionalidade no ambiente hospitalar pode reduzir custos e melhorar a produção do cuidado ${ }^{19}$.

$\mathrm{Na}$ D2 observou-se que os profissionais dentro da faixa etária de 36 a 45 anos, referiram uma percepção discretamente mais positiva entre as demais faixas. Notou-se também que houve uma conformidade entre as categorias profissionais, uma percepção positiva, quanto ao PEP como um recurso estimulador para o trabalho interprofissional.

A interdisciplinaridade é apontada mundialmente como estratégia para reconfiguração da assistência à saúde. Pois, promove uma parceria entre os profissionais de saúde de diferentes campos do conhecimento, o que conduz a abordagem participativa, colaborativa e coordenada de tomada de decisão compartilhada em torno da saúde ${ }^{19}$

AD3 versou sobre a percepção dos profissionais quanto à introdução de novas TIC em saúde como um processo estimulador da EPS na instituição.Nas asserções 10, 14 e 16 observou-se uma compreensão positiva dos respondentes quanto à qualificação de uso do PEP como uma estratégia para o desenvolvimento de um processo de EPS.

A EPS vem caminhando junto com as novas ferramentas tecnológicas, contribuindo para a melhoria dos processos educacionais na área de saúde e para a prática assistencial dos profissionais $^{5,17}$.

Ainda na D3 notou-se uma percepção em zona intermediária da categoria profissional médica a respeito da interação das TIC com desenvolvimento da EPS. Apesar das Diretrizes Curriculares Nacionais do curso de graduação em medicina instituirem a EPS como eixo central para o desenvolvimento dos processos educativos em saúde, muitos médicos não foram formados nesta perpectiva $^{20}$.

A D4 abordou a percepção da equipe de saúde referente ao uso do PEP como um instrumento qualificador para assistência à saúde.

Na D4, as asserçôes 12, 15, 17revelaram que os respondentes mostraram-se inclinados à concordância. Apoiando que o uso do PEP contribuipara organização da assistência, agilidade e interação das ações propostas pela equipe, assim como redução de possíveis erros assistenciais. Benefícios já apontados na literatura ${ }^{8-11}$ que afirmam as inúmeras vantagens e possibilidades advindas da utilização do PEP.

Percebe-se na D4 que os profissionais que 
relataram ter estímulo ao uso de TIC na graduação/ formação, tiveram uma percepção mais positiva em relação aos que não tiveram contato durante a formação acadêmica.

A presença de novas tecnologias enquanto coadjuvante do processo de ensino-aprendizagem, direciona a construção de modelos diferenciados de técnicas de ensino e incentivar o aluno a utilizar recursos tecnológicos ${ }^{17,18}$.

É compreendido, portanto, a importância do serviço de EC como estratégia para o desenvolvimento de recursos humanos nas organizações hospitalares. Assim, como o programa educacional deverá ser dinâmico e interdisciplinar, bem como promover o uso eficiente de tecnologias no ambiente de trabalho.

Em última análise a grande questão de um processo educativo em saúde é a geração de um cuidado integral qualificado. As TIC na saúde não podem adquirir maior relevância que os profissionais que a usam nem tão pouco substituir as relações interpessoais.

Salienta-se a necessidade das instituições hospitalares cenários importantes de prática e formação profissional, a adotarem políticas e programas de EC no âmbito das TIC, no sentido de contribuir positivamente para a qualificação cotidiana de seus profissionais e espaço de reflexão para uso efetivo de tecnologias em saúde.

Como limitação do estudo, deve-se considerar que a amostra utilizada para a realização da pesquisa representa uma pequena parcela da realidade dos hospitais brasileiros, os resultados representam um contexto específico. Como recomendação para estudos futuros, propõe-se que se amplie a amostra utilizada.

Por fim, considera-se a necessidade de maiores estudos acerca do assunto, especificamente, em relação à utilização das TIC na educação permanente dos profissionais da área da saúde, visando à melhoria dos processos assistenciais.

\section{CONCLUSÃO}

Podemos concluir que os participantes reconheceram o programa de EC da instituição como um espaço que promove a construção de significados relacionados ao uso de novas tecnologias no campo da saúde.

O estudo revelou que o desenvolvimento de ações educativas voltadas ao uso de tecnologias em saúde é fundamental para a qualificação e compreensão dos profissionais quanto ao uso em seu cotidiano.

A partir dos dados analisados foi evidenciado pelos respondentes que as atividades desenvolvidas pelo serviço de EC geraram algumas mudanças no processo de trabalho, quando inferiram positivamente que as ações favoreceram a interprofissionalidade.

Ficou demonstrada no estudo a necessidade de adequação da carga horária dos treinamentos, assim como as ações educativas precisam transpassar o processo de trabalho, para submergir a compreensão do uso de tecnologias no processo de cuidar.

\section{REFERÊNCIAS}

1. Krummenauer EC, Machado JAA, Kautzmann AE, Ritta CM, Haas F, Carneiro M. Educação continuada: Uma ferramenta para a segurança do cuidado. RevEpidemiolControllnfect. 2014;4(3):221-22.

2. Pinochet LHC, Lopes AS, Silva JS. Inovações e tendências aplicadas nas tecnologias de informação e comunicação na gestão da saúde. Rer. RGSS. 2014;3(2):11-9.

3. Somera EAS, Somera Jr R, Rondina JM. Uma proposta da andragogia para a educação continuada na área da saúde. ArqCiênc Saúde. 2010;17(2):102-8.

4. Azevedo IC, Silva GWS, Vale LD, Santos QG, Cassiano AN, Morais IF, Valença CN.Educação continuada em enfermagem no âmbito da educação permanente em saúde: revisão integrativa de literatura. Revista Saúde e Pesquisa. 2015;8(1):131-40.

5. Campos KFC, Sena RR, Silva KL. Educação permanente nos serviços de saúde. Esc Anna Nery 2017;21(4):e20160317.

6. Pinochet LHC, Lopes AS, Silva JS. Inovações e tendências aplicadas nas tecnologias de informação e comunicação na gestão da saúde. RGSS, Revista de Gestão em Sistemas de Saúde. 2014;3(2):11-19.

7. Lorenzetti J, Trindade LL, Pires DEP, Ramos FRS. Tecnologia, inovação tecnológica e saúde: uma reflexão necessária. Texto Contexto Enferm, 2012;21(2):432-9.

8. Gonçalves JPP, Batista LR, Carvalho LM,Oliveira MP, Moreira KS, Leite MTS. Prontuário Eletrônico: uma ferramenta que pode contribuir para a integração das Redes de Atenção à Saúde. Saúde em Debate. 2013;37(96):43-50.

9. Lourenço LG, Ferreira CJ. Implantação do prontuário eletrônico do paciente no Brasil. Enfermagem Brasil. 2016;15(1):44-53

10. Jenal S, Évora YDM. Desafio da implantação do prontuário eletrônico do paciente J. Health Inform. 2012;4(Número Especial - SIIENF 2012): 216-9. 
11. Lahm JV, Carvalho DR. Prontuário eletrônico do paciente: avaliação de usabilidade pela equipe de enfermagem. CogitareEnferm. 2015;20(1):38-44.

12. Souza AC, Alexandre NMC, Guirardello EB. Propriedades psicométricas na avaliação de instrumentos: avaliação da confiabilidade e da validade. Epidemiol. Serv. Saude.2017;26(3):649-59.

13. Wakita T, Ueshima N, Noguchi H. Psychological distance between categories in the Likert Scale: comparing different numbers of options. EPM. 2012;72(4):533-46.

14. Bucchi SM, Mira VL. Reelaboração do treinamento admissional de enfermeiros de Unidade de Terapia Intensiva. RevEscEnferm USP, 2010;44(4):1003-10.

15. Costa DB, Vannuchi MTO, Haddad MCFL, Cardoso MGP Silva LG, Garcia SD. Custo de educação continuada para equipe de enfermagem de um hospital universitário público. Rev. Eletr. Enf. 2012;14(2):257-66.

16. Grohmann ZM, Battistella LF. Homens e mulheres "aceitam" de maneira diferente? impacto do gênero no modelo (expandido) de aceitação da tecnologia - TAM. Inf\& Soc. 2011;21(1):175-189.

17. Rezende R, Oliveira JEE, Friestino JKO. A educação permanente em enfermagem e o uso das tecnologias: uma revisão integrativa. R. Interd. 2017;10(1):190-99.
18. PissaiaLF, Costa AEK, RehfeldtMJHe, Moreschi C Tecnologia educacional no processo de formação de enfermeiros. Cinergis. 2017;18(3):185-89.

19. Ellery AEL. Interprofissionalidade na Estratégia Saúde da Família: condições de possibilidade para a integração de saberes e a colaboração interprofissional. Interface Comun Saúde Educ. 2014; 18(48):213-4.

20. Brasil. Ministério da Educação. Conselho Nacional de Educação. Câmara de Educação Superior. Resolução do Diário Oficial da União. Brasília, CNE/CES de 7/11/ 2001. Institui diretrizes curriculares nacionais do curso de graduação em medicina.

\section{CORRESPONDENCIA}

Nome: Rosane Barreto Cardoso

Endereço: Av. Embaixador Abelardo Bueno, 3000 - Barra da Tijuca.

Rio de Janeiro - Rio de Janeiro - Brasil

CEP: 22775-040

Email: rosane.bcardoso@gmail.com 